Manuelle Medizin 2019·57:389-390

https://doi.org/10.1007/s00337-019-00616-x

(C) Springer Medizin Verlag GmbH, ein Teil von Springer Nature 2019

\author{
K.-St. Delank' $\cdot$ R. Kayser \\ 'Department für Orthopädie, Unfall- und Wiederherstellungschirurgie, Universitätsklinikum Halle, Halle \\ (Saale), Deutschland \\ ${ }^{2}$ MEDIAN Klinik Wismar · Abteilung Orthopädie, MEDIAN Kliniken GmbH, Wismar, Deutschland
}

\title{
Lumbale Instabilität
}

Die funktionelle Integrität der Wirbelsäule bzw. des Bewegungssegments wird mit den Begriffen „Stabilität“ bzw. „Instabilität" formuliert, ohne dass diese bis heute zweifelsfrei definiert sind.

Eine Definition von Gertzbein (1985) beschreibt die Instabilität des Bewegungssegments als pathologische Beweglichkeit oberhalb normaler Grenzen bei normaler Krafteinleitung.

Eine den komplexen Aufgaben der Wirbelsäule gerechter werdende Definition wurde von White und Panjabi (1990) vorgelegt. Die Autoren definieren Instabilität als das Unvermögen der Wirbelsäule, ihr Bewegungsmuster unter physiologischen Bedingungen so aufrechtzuerhalten, dass ein neurologisches Defizit, Schmerzen oder eine morphologische Verformung auftreten können.

Die Beweglichkeit kann dabei qualitativ verändert sein, d.h. in Form sog. pathologischer gekoppelter Bewegungen, oder aber quantitativ durch eine pathologische Veränderung des Bewegungsumfangs.

Die sehr komplexe und oft nicht genau feststellbare Kausalität von Wirbelsäulenbeschwerden hat zur Folge, dass der wenig konkret definierte Begriff der „Instabilität“ häufig eingesetzt wird, ohne dass die Bedeutung der mechanischen und kinematischen Veränderungen im individuellen Fall bekannt ist.

Über die Problematik einer Begriffsbeschreibung hinaus geht die Frage, welche additive mechanische Stabilität des Bewegungssegments durch eine therapeutische Maßnahme benötigt wird, um eine bestimmte Symptomatik zu lindern; oder aber anders formuliert, welcher Grad der Instabilität ist zu akzeptieren, ohne dass ein neurologisches
Defizit, Schmerzen oder morphologische Verformungen auftreten können.

Das zentrale Problem für die Beantwortung dieser Frage liegt in der Tatsache, dass zwar die morphologischen Veränderungen an der Wirbelsäule mithilfe der modernen bildgebenden Verfahren hervorragend dargestellt, die funktionellen Veränderungen aber bislang nicht ausreichend reproduzierbar beurteilt werden können.

Rückenschmerzen haben bekanntermaßen eine sehr hohe gesundheitspolitische und gesellschaftliche Relevanz. Fast jeder Mensch kennt Episoden mit Rückenschmerzen, häufig bilden sich diese spontan zurück, aber 30-50\% der Bevölkerung leiden an chronischen Rückenschmerzen. Die Diagnostik und Behandlung dieser Patienten wird einerseits von Ärzten und Therapeuten mit großer Erfahrung im konservativen Bereich durchgeführt, andererseits bietet auch die Wirbelsäulenchirurgie erfolgversprechende operative Therapieansätze an. An dieser „Schnittstelle operative/konservative Wirbelsäulentherapie" treffen oftmals sehr unterschiedliche Sichtweisen der Behandler aufeinander.

\section{》) Die adäquate Versorgung der Patienten gelingt nur mit einem interdisziplinären Ansatz}

Aus Sicht der manuellen Medizin ist es heute etabliertes klinisches Wissen, dass strukturelle segmentale Instabilität kompensatorische Funktionsstörungen generieren kann. Zudem haben Techniken der muskulären Stabilisierung einen festen Platz in den heute angewendeten Behandlungsprogrammen. Hier ist die 
Schnittmenge zur schwer zu fassenden Mikroinstabilität offensichtlich und fördert den Gedanken der manuellen Diagnostik von Dekompensationsmustern jenseits der bildgebend fassbaren Veränderungen.

Das vorliegende Themenheft befasst sich mit dieser komplexen Problematik und gibt einen Überblick über die verschiedenen Interpretationen der „lumbalen Instabilität" und die möglichen therapeutischen Konsequenzen. Ausgewiesene Experten teilen ihre Erfahrungen mit der Leserschaft, liefern wertvolle Praxistipps und diskutieren aktuelle Literaturergebnisse.

Dabei wird eindrucksvoll sichtbar, dass die adäquate Versorgung dieser Patienten nur mit einem interdisziplinären Ansatz gelingen kann.

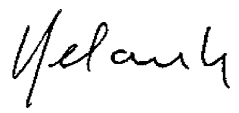

Prof. Dr. K.-St. Delank<smiles>CCCCC(C)C(C)(C)C</smiles>

Prof. Dr. R. Kayser

\section{Korrespondenzadresse}

\section{Prof. Dr. K.-St. Delank}

Department für Orthopädie, Unfall- und Wiederherstellungschirurgie, Universitätsklinikum Halle

Ernst-Grube-Straße 40, 06120 Halle (Saale), Deutschland

stefan.delank@uk-halle.de

\section{Prof. Dr. R. Kayser}

MEDIAN Klinik Wismar · Abteilung Orthopädie, MEDIAN Kliniken GmbH

Ernst-Scheel-Straße 28, 23968 Wismar,

Deutschland

ralph.kayser@median-kliniken.de

Interessenkonflikt. K.-S. Delank und R. Kayser geben an, dass kein Interessenkonflikt besteht.

\section{Wie Rückenschmerzen besser diagnostiziert werden können}

\section{Fünf von sechs Wissenschaftspreisen des Bundesverbandes der selbstständigen Physiotherapeuten gehen an Absolventinnen und Absolventen der Hochschule Osnabrüick.}

Laut DAK-Gesundheitsreport leiden drei Viertel aller Berufstätigen mindestens einmal im Jahr unter Rückenschmerzen. Um die Defizite zu identifizieren, nutzen viele Physiotherapeutinnen und Physiotherapeuten sogenannte Motor Control Tests (MCT). Doch bislang ist unklar, welche Testbestandteile ideal sind, um unspezifische Rückenschmerzen sicher zu diagnostizieren. Hier setzt die Masterarbeit von Christoph Biele an, für die der Absolvent des berufsbegleitenden Masterstudiengangs "Muskuloskelettale Therapie“ jetzt mit dem 1. Platz des Wissenschaftspreises des Bundesverbands der selbständigen Physiotherapeuten (IFK) ausgezeichnet worden ist. Dieser ist mit 1.500 Euro dotiert.

Der IFK ehrt seit 2006 jedes Jahr die besten Bachelor- und Masterarbeiten in Bezug auf ein physiotherapeutisches Thema. Die Arbeiten werden von acht Jurymitgliedern unabhängig voneinander beurteilt. In diesem Jahr gehen gleich fünf der sechs Preise an Absolventinnen und Absolventen der Hochschule Osnabrück.

Preisträger Biele hat rund 130 Probandinnen und Probanden mit und ohne Rückenschmerzen untersucht und eine bislang aus sechs Bewegungstests bestehende sogenannte Testbatterie auf elf Tests erweitert. "Diese haben in meinen Untersuchungen ein Maximum an diagnostischer Genauigkeit gebracht und sind dazu zeitökonomisch sinnvoll", so Biele. "Mich reizt das Thema schon lange, da ich bereits seit vielen Jahren in Physiotherapiepraxen arbeite und viele Berührungspunkte mit Rückenpatienten habe."

Auch die besten Bachelorarbeiten kommen aus Osnabrück

In der Kategorie „Bachelorarbeiten - Klinisch/Experimentell“ hat sich die Jury ebenfalls für Abschlussarbeiten aus Osnabrück ausgesprochen. Lena Lingemann und Svenja Nilsson teilen sich den ersten Preis in Höhe von 1.000 Euro. Die beiden Absolventinnen des Studiengangs „Ergotherapie, Physiotherapie dual“ haben Sängerinnen und Sänger mit und ohne Stimmproblemen untersucht. „Wir wollten herausfinden, ob sich die beiden Gruppen in der Kehlkopffunktion oder in ihrer Haltungs- und Bewegungskontrolle der Nacken-, Kiefer- und Gesichtsregion unterscheiden", so Nilsson. Dazu haben sie unter anderem Bewegungstests der Nacken, Kiefer- und Gesichtsregion durchgeführt und die Kehlköpfe manuell auf Schmerzen und Widerstände untersucht. „Wir haben bislang keinen direkten Zusammenhang feststellen können, es würde sich aber lohnen, die Versuche mit einer anderen Gruppenzusammensetzung zu wiederholen", so die Preisträgerinnen.

Über weitere Preise können sich die Osnabrücker Absolventinnen und Absolventen Daniela von Piekartz-Doppelhofer (2. Platz beste Masterarbeit), Bettina Veit (2. Platz beste Bachelorarbeit - Klinisch/Experimentell) sowie Lukas Reineke und Julius Schwiddessen (2. Platz beste Bachelorarbeit - Literatur/Konzept) freuen.

Quelle: Hochschule Osnabrück, http://www.hs-osnabrueck.de 